\title{
Prevention of peritoneal-dialysis-related infections -antibacterial honey might not be the solution
}

$\mathrm{T}$ opical application of medical-grade antibacterial honey to the catheter exit-site is not more effective than standard exit-site care with additional intranasal mupirocin in carriers of Staphylococcus aureus for the prevention of infections related to peritoneal dialysis. This new finding from the open-label HONEYPOT trial by David Johnson and colleagues was recently published in The Lancet Infectious Diseases.

"Honey obtained from bees fed on Leptospermum plant species has antibacterial properties and has been successfully used for wound healing and the eradication of infection," explains researcher Sunil Badve, an author of the new paper. "One advantage of honey over antibiotics is that it does not induce antibacterial resistance." Johnson and colleagues previously demonstrated comparable infection rates and reduced rates of antibacterial resistance with exitsite application of antibacterial honey (three times per week) versus mupirocin in patients with end-stage renal disease (ESRD) receiving haemodialysis. Rates of local skin reactions were low (2\%) in both groups and no participants discontinued treatment.
To assess the efficacy
of antibacterial honey
for the prevention of discontinued treatment.
To assess the efficacy
of antibacterial honey
for the prevention of peritoneal-dialysisrelated infections, Johnson and his co-workers recruited 371 patients with ESRD who were undergoing peritoneal dialysis at 26 centers in Australia and New Zealand. Patients were randomly assigned to daily self-application of antibacterial honey to the exit site in addition to standard exitsite care $(n=186)$ or to standard exit-site care $(n=185)$ with additional intranasal mupirocin prophylaxis in those who tested positive for nasal carriage of $S$ aureus. The primary end point was time to first peritoneal-dialysis-related infection (a composite of exit-site infection, tunel infection and peritonitis).

The researchers report no significant difference in the primary end point between the honey and control groups (mean of 16 months versus 17.7 months, unadjusted hazard ratio [HR] 1.12, 95\% CI $0.83-1.51, P=0.47)$. The incidences of severe adverse events and of death were also similar in the two groups. Mupirocin resistant $S$. aureus was detected in two of 27 patients with infections in the control group and in none of the 34 patients with infections in the honey group. However, this difference was not statistically significant. More patients in the honey group than in the control group discontinued treatment $(67 \%$ versus $50 \%$ ) and $6 \%$ of patients in the honey group withdrew from the study because of local skin reactions. Johnson et al. suggest that the higher rates of skin reactions and treatment discontinuation in the current study than in their previous trial of antibacterial honey in patients on haemodialysis might be related to methodological differences, including differences in catheter type and frequency of honey application.

In their subgroup analysis, the researchers found that in patients with diabetes mellitus, use of antibacterial honey compared with standard care was associated with significantly increased risks of peritoneal-dialysis-related infections (HR 1.85, 95\% CI 1.05-3.24, $P=0.03$ ) and peritonitis (HR 2.25, 95\% CI 1.16-4.36, $P=0.002)$. However, Badve emphasizes that caution should be exercised when interpreting this analysis, which involved only 115 patients. He states that the observed result could be due to a type 1 statistical error and points out that the presence of diabetes perse was not associated with an increased risk of infection. "We cannot definitely conclude that honey was harmful in patients with diabetes and this subgroup analysis should be regarded as hypothesis-generating only," he says. Badve suggests that in future studies of interventions to prevent peritonealdialysis-related infections, randomization of patients to treatment groups should include stratification for diabetes.

"While the fact that honey doesn't contribute to antibacterial resistance makes it an attractive option for preventing infection at wound sites, our results suggest that honey cannot be routinely recommended for the prevention of infections related to peritoneal dialysis," concludes Johnson. "Not only do our results show that honey doesn't work any better than standard exit-site care and additional nasal mupirocin for nasal carriage of $S$. aureus in protecting peritoneal dialysis patients from infection, we had a high rate of withdrawal from the study in the honey group, usually at the request of the patient or physician. This suggests that patients may have found the daily application of honey to the wound site uncomfortable or inconvenient."

\section{Ellen F. Carney}

\footnotetext{
Original article Johnson, D. W. et al. Antibacterial honey for the prevention of peritoneal-dialysis-related infections (HONEYPOT): a randomised trial. Lancet Infect. Dis. doi:10.1016/S1473-3099(13)70258-5

Further reading Johnson, D. W. et al. Randomized, controlled trial of topical exit-site application of honey (Medihoney) versus mupirocin for the prevention of catheter-associated infections in hemodialysis patients. J. Am. Soc. Nephrol. 16, 1456-1462 (2005)
} 